\title{
Quasi-Analytical Static Solution of the Boxed Microstrip Line Embedded in a Layered Medium
}

\author{
F. Medina, Member, IEEE, and M. Horno, Member, IEEE
}

\begin{abstract}
In this paper, a quasi-analytical method is presented to carry out the quasi-TEM study of a microstrip line embedded in a general layered substrate with rectangular enclosure. Electric walls, magnetic walls and periodic boundary conditions are considered. The analysis is based on the spectral domain formulation and the use of a proper expansion of the free charge distribution (Chebyshev polynomials with edge condition). Two different approaches are proposed to speed up the evaluation of the spectral series in such a way that only a few spectral terms must be retained in the numerical computations of the mentioned series. The propagation parameters and the charge distribution are obtained with extreme accuracy in fractions of one second on a personal computer.
\end{abstract}

\section{INTRODUCTION}

$\mathrm{M}$ ICROSTRIP is undoubtedly one of the most popular ransmission lines used in modern microwave technology. The propagation characteristics of this transmission line have been computed by using a variety of numerical and analytical tools during the last three decades. However, analytical or quasi-analytical solutions have only been provided for a limited number of cases. Many of these solutions, based on conformal mapping techniques, can only be applied to certain particular geometries (for example [1]-[3]). Some other numerically efficient methods involving a high degree of analytical preprocessing and/or physical insight in the nature of the problem can also be found in the literature. The WienerHopf method [4], singular integral equation (SIE) [5], [6], matched asymptotic expansions [7] and other techniques [8], [9] are good pieces of this type of work. Nevertheless, relevant but simple configurations were considered by those authors.

Recently, several authors have focused their attention on the exact analysis of different microstrip structures and/ or on the improvement of the computational aspects of classical numerical techniques. Kretch and Collin [10] have used a very efficient perturbation-iteration method for the full-wave analysis of a microstrip line printed on an anisotropic dielectric substrate. Fikioris et al. [11] have presented an extremely accurate quasi-TEM study of the boxed microstrip line printed on a single lossless isotropic

Manuscript received Augus1 11, 1991; revised February 18, 1992. This work was supported by the DGICYT, Spain, Project No. TIC $91-1018$

The authors are with the Universidad de Sevilla, Facultad de Fisica Departamento de Electronica y Electromagnetismo, 41012, Sevilla, Spain. IEEE Log Number 9201711. substrate. In the latter paper, the authors have solved a Carleman-type integral equation whose kernel (Green's function) has been very efficiently computed by using three alternative rather involved approaches [12]. Uchida et al. [13] and Homentcovschi [14] have obtained dynamic and quasistatic solutions for the open microstrip problem on a single layer substrate by employing proper numerical tools in the spectral domain. Efficient spacedomain formulations have also been reported in [15] (quasi-TEM) and [16] (full-wave).

This work shares the aim with the previously refered to papers. The structure considered in this paper is the microstrip line embedded in a layered configuration with arbitrary rectangular boundary conditions (see Fig. 1(a)). This problem can not be exactly solved but, as will be shown, can be numerically treated in an extremely efficient way by making use of a suitable analytical preprocessing. Owing to the presence of the layered medium, the problem is conveniently formulated by means of the well known spectral domain analysis (SDA). The Green's function (static or dynamic) can be easily computed in the spectral domain using appropriate existing algorithms which can handle anisotropic and lossy materials. In this paper we will restrict ourselves to the quasi-TEM model, since the essential features of the techniques described in the paper should be readily extended to full-wave formulations. The application of the Ritz and Galerkin methods in the spectral domain leads to a system of linear equations whose entries are slowly convergent series. Although the straightforward application of the SDA makes it possible to obtain results which are accurate enough for many practical purposes, convergence is not satisfactory. Moreover, charge distributions can not be accurately computed with reasonable computational effort. In fact, erroneous results are likely to be obtained in certain critical cases. Fortunately, if physically suitable basis functions are used and the asymptotic tails of the series involved in the computations are analytically added, the power of the SDA can be meaningfully enhanced. Drastic improvement of accuracy and CPU savings can be achieved in this way. Two different techniques are proposed in this paper to enhance the SDA with a reasonable degree of generality and relatively low analytical complexity. The first method makes use of the residue calculus technique to speed up the convergence of the series. 


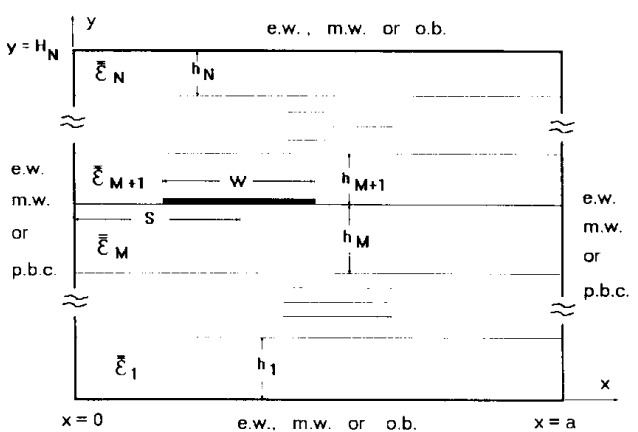

(a)

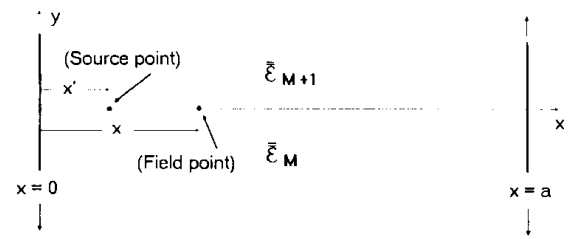

(b)

Fig. 1. (a) Cross section of the generalized boxed microstrip line. $x=0$ and $x=a$ can be e.w., m.w. or p.b.c. $y=0$ and $y=H_{N}$ can be e.w., m.w. or o.b. (b) Asymptotic microstrip problem. The spatial domain Green's function for this problem is in Table II.

The second method is based on the consideration of an appropriate auxiliar asymptotic problem (see Fig. 1(b)) whose Green's function is analytically known in the spatial domain. Convolution integrals and inner products are also quasi-analytically calculated. Extremely accurate results (for both propagation characteristics and charge distribution) are obtained with this method in fractions of one second on a PC computer, thus making it appropriate for CAD purposes. The reliability of the computer codes has been exhaustively tested by comparing with highly accurate data available in the literature for single layer configurations.

Single and symmetrically coupled strips are considered in this paper, but the method is expected to be extended to multistrip systems. The method should also be useful to accelerate the computations when a dynamic model is used, since convergence problems are mainly associated with the quasi-TEM limit of the dynamic problem. Anyway, certain additional improvements are feasible in the dynamic case. It should be noted that although a closed structure is considered, open microstrip can be treated with slight modifications by considering spectral integration instead of series addition. In fact, for open structures, a third alternative was explained in [18].

\section{Outline of the Problem}

The general microstrip line analyzed in this paper is shown in Fig. 1(a). A perfectly conducting, $w$ wide, zerothickness strip is embedded in a layered medium composed of lossless/lossy isotropic/anisotropic slabs. The whole structure is assumed to be placed inside a rectangular enclosure bounded by the planes $x=0, x=a, y=$ $0, y=H_{N}$. The planes $y=0, y=a$ can be electric walls (e.w.), magnetic walls (m.w.) or open boundaries (o.b.). The planes $x=0, x=a$ can be e.w., m.w. or periodic boundary conditions (p.b.c.). It is obvious that a wide variety of single and symmetrically coupled strip configurations are particular cases of the structure appearing in Fig. 1(a).

The quasi-TEM analysis of the transmission line shown in Fig. 1(a) reduces to solving the bidimensional Laplace's problem in the $x-y$ plane. Since an arbitrary number of layers must be considered, it is more appropriate to work in the spectral domain than in the spatial domain. The spectral static Green's function (SSGF), $\tilde{G}_{M}$, is readily computed by using the method in [17] (lossy and/or magnetic materials can also be accommodated by following [18]). For simplicity purposes, we will restrict ourselves in the exposition to the dielectric lossless case. Anyway, the results can be easily applied to lossy and/or magnetic cases, since differences mainly arise from the Green's function rather than from the analytical and computational aspects, which are basically the same as those treated in this paper. Although the spectral domain analysis of the microstrip line has been widely discussed in the literature, a brief summary will be given next in order to put this work in context. The relationship between the Fourier transforms of the surface charge distribution on the strip, $\tilde{\sigma}$, and the potential distribution at the plane where the strip is printed, $\tilde{\phi}$, is given by

$$
\tilde{G}_{M}\left(\alpha_{n}\right) \cdot \tilde{\sigma}\left(\alpha_{n}\right)=\tilde{\phi}\left(\alpha_{n}\right)
$$

where $\alpha_{n}$ stands for the Fourier variable. The proper definition of $\alpha_{n}$ and the Fourier transform depends on the nature of the boundary conditions in $x=0$ and $x=a$. The suitable choices at four different common situations are summarized in Table I. The problem posed by (1) can be solved by using the Ritz method (in lossless cases) [17] or Galerkin method [18]. In both cases, the unknown free surface charge distribution, $\sigma(x)$, is expanded into a set of suitable basis functions whose coefficients are computed by requiring that the electrostatic energy per unit length is a minimum (Ritz method) or that the potential on the strip is constant (Galerkin method). Both techniques lead to a set of linear equations whose unknowns are the expansion coefficients. In order to prevent the size of this system from being too large, it is crucial to choose the basis functions in an adequate way. Concerning this subject, it has been conveniently stablished in the literature that a particularly suitable set of basis functions are the Chebyshev polynomials weighed by the Maxwell edge condition. The surface charge distribution can then be written as follows:

$$
\begin{aligned}
\sigma(x)= & \sum_{q=0}^{n f} a_{q} \sigma_{q}(x) \\
= & \frac{2}{\pi w} \frac{1}{\sqrt{1-\left(\frac{x-s}{w / 2}\right)^{2}}} \sum_{q=0}^{n f} a_{q} T_{q}\left(\frac{x-s}{w / 2}\right) ; \\
& s-w / 2 \leq x \leq s+\omega / 2
\end{aligned}
$$


TABLE 1

Fourier Variable (F.V.), Fourier Transform Definition (F.T.D.) and Fourier TRANSFORMS OF BaSIS FunCTIONS IN (2) (F.T.B.F.) FOR Different Combinations of Lateral Boundary Conditions. Electric $W_{A L L}=$ e.w.; MaGnetiC $W_{A L L}=m . w .$, PERIOdic Boundary CONDITION $=$ p.b.c.

\begin{tabular}{|c|c|c|}
\hline & $\begin{array}{l}x=0: \text { e.w. } \\
x=\text { a: e.w. }\end{array}$ & $\begin{array}{l}x=0: \text { m.w. } \\
x=\text { a: e.w. }\end{array}$ \\
\hline F.V & $\alpha_{n}=\frac{n \pi}{a}$ & $\alpha_{n}=\frac{(2 n-1) \pi}{2 a}$ \\
\hline $\begin{array}{l}\text { F.T.D. } \\
\left(\tilde{\sigma}\left(\alpha_{n}\right)\right)\end{array}$ & $\int_{0}^{a} \sigma(x) \sin \left(\alpha_{n} x\right) d x$ & $\int_{0}^{a} \sigma(x) \cos \left(\alpha_{n} x\right) d x$ \\
\hline \multirow[t]{2}{*}{$\begin{array}{l}\text { F.T.B.F. } \\
p \text { even } \\
p \text { odd }\end{array}$} & $\begin{array}{l}J_{p}\left(\alpha_{n} w / 2\right) \\
(-1)^{p / 2} \sin \left(\alpha_{n} s\right) \\
(-1)^{(p+1 s / 2} \cos \left(\alpha_{n} s\right)\end{array}$ & $\begin{array}{l}J_{p}\left(\alpha_{n} w / 2\right) \cdot \\
(-1)^{(p-1) / 2} \cos \left(\alpha_{n} s\right) \\
\left(-1^{i p+1) / 2} \sin \left(\alpha_{n} s\right)\right.\end{array}$ \\
\hline & $\begin{array}{l}x=0: \text { p.b.c. } \\
x=a: \text { p.b.c. }\end{array}$ & $\begin{array}{l}x=0: \mathrm{m} . \mathrm{w} \\
x=a: \mathrm{m} . \mathrm{w}\end{array}$ \\
\hline F.V. & $\alpha_{n}=\frac{2 n \pi}{a}$ & $\alpha_{n}=\frac{n \pi}{a}$ \\
\hline $\begin{array}{l}\text { F.T.D. } \\
\left(\tilde{\sigma}\left(\alpha_{n}\right)\right)\end{array}$ & $\int_{0}^{a}(x) \exp \left(j \alpha_{n} x\right) d x$ & $\int_{0}^{a} \sigma(x) \exp \left(j \alpha_{n} x\right) d x$ \\
\hline $\begin{array}{l}\text { F.T.B.F. } \\
p \text { even } \\
p \text { odd }\end{array}$ & $\begin{array}{l}J_{p}\left(\alpha_{n} w / 2\right) \\
(-1)^{p} \exp \left(j \alpha_{n} s\right) \\
(-1)^{p} \exp \left(j \alpha_{n} s\right)\end{array}$ & $\begin{array}{l}J_{p}\left(\alpha_{n} w / 2\right) \\
(-1)^{p / 2} \cos \left(\alpha_{n} s\right) \\
(-1)^{(p+1) / 2} \sin \left(\alpha_{n} s\right)\end{array}$ \\
\hline
\end{tabular}

When the basis functions in (2) are employed and the Galerkin's method is applied to (1), the following system of linear equations for the coefficients $a_{q}$ is obtained:

$A_{p, q} \cdot a_{q}=B_{p}$ where $p, q=0, \cdots, n f$

$A_{p, q}=\sum_{n=1}^{\infty} \tilde{\sigma}_{p}^{*}\left(\alpha_{n}\right) \cdot \tilde{G}_{M}\left(\alpha_{n}\right) \cdot \tilde{\sigma}_{q}\left(\alpha_{n}\right) ; \quad B_{p}=\delta_{0, p}$

where $\tilde{\sigma}_{q}$ stands for the Fourier transform of the basis functions in (2) (which are tabulated in Table I) and $\delta_{0, p}$ is the Kronecker delta. It should be pointed out that the term $n=0$ must be added in (4) when necessary and that only even order basis functions should be considered in the case of lateral periodic boundary conditions.

The computational effort is mainly spent in the calculation of the entries of the Galerkin (or Ritz) matrix, $A_{p . q}$. The entries are slowly convergent series involving products of the Fourier transforms of the basis functions and the SSGF. Owing to the wide spectrum of these functions, the truncation of the spectral series may give place to serious errors in case a suitable technique to speed up the computations is not employed. This fact is particularly true for narrow and/or strongly coupled strips, specially when the computation of the charge distribution is required. In order to speed up the computation of (4), these series are split in the following way:

$$
A_{p, q}=\sum_{n=1}^{\infty} \tilde{\sigma}_{p}^{*}\left[\tilde{G}_{M}-\tilde{G}_{M}^{u s}\right] \tilde{\sigma}_{q}+S_{p, q}
$$

$$
S_{p, q}=\sum_{n=1}^{\infty} \tilde{\sigma}_{p}^{*} \tilde{G}_{M}^{d s} \tilde{\sigma}_{q} ; \quad \tilde{G}_{M}^{a s}=\frac{1}{\alpha_{n} \epsilon_{e q}} ; \quad \epsilon_{e q}=\frac{\epsilon_{M}^{e q}+\epsilon_{M+1}^{e q}}{2}
$$

where $\epsilon_{i}^{e q}$ is the permittivity (or the "equivalent" permittivity for anisotropic [17] or magnetic cases [18]) of the $i$ th layer. Since $\tilde{G}_{M}$ exponentially reachs its asymptotic limit, $\tilde{G}_{M}^{a s}$, the first term in (5) typically converges within a few Fourier terms. However, the convergence of $S_{p, q}$ is extremely slow. Fortunately, analytical or quasi-analytical expressions can be provided for $S_{p, q}$ when the basis functions in (2) are used. This will be shown in the following two sections.

\section{First Method: Computation of Tails by Residue Calculus Technique}

The first technique to compute efficiently $S_{p, q}$ is based on transforming (6) into very quick convergent power series. The general term of the series $S_{p, q}$ involves products of integer order first kind Bessel functions $\left(J_{p}\right)$ and, eventually, trigonometric functions. Two kinds of series must be calculated:

$$
S_{p . q}^{\prime}=\sum_{n=1}^{\infty} \frac{J_{p}(\alpha n) J_{q}(\alpha n)}{n} \cdot \begin{cases}\cos (c n) ; & p+q \text { is even } \\ \sin (c n) ; & p+q \text { is odd }\end{cases}
$$

$S_{p, q}^{\prime \prime}=\sum_{n=1}^{\infty} \frac{J_{p}(\alpha n) J_{q}(\alpha n)}{n} ; \quad p+q$ is even

with $\alpha=(\pi w / 2 a)$ or $(\pi w / 4 a)$ and $c=(2 \pi s / a)$ or $(\pi s / a)$. These series can be viewed as the addition of the residue of certain complex functions. This is the basis of the first summation technique reported in this work.

\section{A. Computation of $S_{p . q}^{\prime}$}

Let us consider the complex function:

$$
f(z)=\frac{J_{p}(\alpha z) J_{q}(\alpha z) \exp (j c z)}{z[\exp (j 2 \pi z)-1]} ; \quad p+q \geq 1
$$

If (9) is integrated in the complex $z$-plane along the closed contour shown in Fig. 2(a), the application of the Cauchy theorem leads to the following expression:

$$
\begin{aligned}
S_{p, q}^{\prime}= & \left\{\begin{array}{ll}
(-1)^{(p+q / 2)} \\
(-1)^{(p+q-1) / 2}
\end{array}\right\} \int_{0}^{\infty} d y \frac{I_{p}(\alpha y) I_{q}(\alpha y)}{y \sinh (\pi y)} \\
& \cdot \begin{cases}\cosh [(\pi-c) y] ; & p+q \text { is even } \\
\sinh [(\pi-c) y] ; & p+q \text { is odd. }\end{cases}
\end{aligned}
$$

The integrals in (10) involving modified Bessel functions $-I_{p}$-can be now expressed in terms of very quickly convergent power series as it is explained in the Appendix. For most practical purposes, the power series can be viewed as closed form expressions, since very few terms need to be retained in a typical case. Anyway, these formulas are still useful even in those critical cases requiring larger number of terms. 


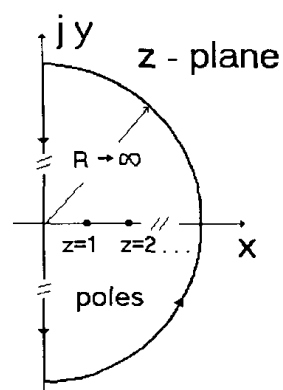

(a)

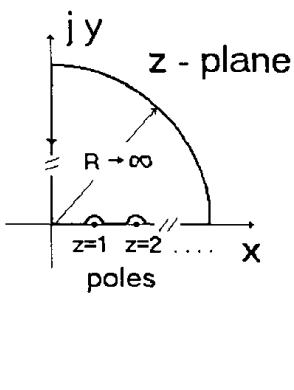

(b)
Fig. 2. Closed paths in the complex plane used in the first method for the evaluation of the spectral series.

Equation (9) cannot be applied to the case $p=0, q=$ 0 due to the existence of a double pole in $z=0$ in this case. In order to overcome this difficulty, let us consider:

$$
\frac{d S_{0,0}^{\prime}}{d \alpha}=-2 \sum_{n=1}^{\infty} J_{0}(\alpha n) J_{1}(\alpha n) \cos (c n)
$$

The series (11) can now be added by using the Cauchy theorem once again. In this case, the complex function to be integrated around the contour shown in Fig. 2(a) must be $z \cdot f(z)$ (where $f(z)$ is obtained by taking $p=0, q=$ 1 in (9)). Acting in this way, the following expression is obtained:

$$
\frac{d S_{0,0}^{\prime}}{d \alpha}=\int_{0}^{\infty} I_{0}(\alpha y) I_{1}(\alpha y) \frac{\sinh [(\pi-c) y]}{\sinh (\pi y)} d y
$$

Then, the right hand term in (12) is transformed into a power series in $\alpha$. This series is integrated with respect to this parameter getting the desired expression for $S_{0,0}^{\prime}$. The final results are shown in Appendix.

\section{B. Computation of $S_{p, q}^{\prime \prime}$}

Let us now consider the complex function:

$g(z)=\frac{J_{p}(\alpha z) J_{q}(\alpha z) \exp (j 2 \pi z)}{z[\exp (j 2 \pi z)-1]} ; \quad p+q$ is even

If (13) is integrated along the closed contour in Fig. 2(b), (8) can be rewritten:

$$
\begin{aligned}
S_{p, q}^{\prime \prime}= & \int_{0}^{\infty} \frac{J_{p}(t) J_{q}(t)}{t} d t+2(-1)^{(p+q / 2)} \\
& \cdot \int_{0}^{\infty} \frac{I_{p}(t) I_{q}(t) \exp \left(-\frac{2 \pi}{\alpha} t\right)}{t\left[1-\exp \left(-\frac{2 \pi}{\alpha} t\right)\right]} d t
\end{aligned}
$$

The first term in the second member is the WeberSchafheitlin integral, which can be found in closed form elsewhere [[19], p. 693]. The second term is reduced to a very quick convergent power series such as that shown in the Appendix. Caution should be taken when $p=q=$ 0 . The same derivation/integration process described in the previous section must be applied here. The final results for this case have been also included in the Appendix.

To sum up, all the asymptotic tails appearing in the spectral domain calculations are very efficiently computed by using the formulas included in this section and in the Appendix. It should be noticed here that the final expressions in the Appendix only involve intrinsic FORTRAN functions-i.e., Bessel functions or other special functions subroutines are not used-resulting in additional CPU time savings.

\section{Second Method: Spatial Domain Computation OF TAILS}

A second procedure of similar numerical efficiency as that explained in the previous section is based on the evaluation of $S_{p, q}$ by using a proper integration in the spatial domain. Making use of Parseval and convolution theorems, (6) can be rewritten in the following way:

$$
\begin{gathered}
S_{p, q}=\int_{\text {strip }} d x \frac{2}{\pi \omega} \frac{T_{p}\left(\frac{x-s}{w / 2}\right)}{\sqrt{1-\left(\frac{x-s}{w / 2}\right)^{2}}} \int_{\text {strip }} d x^{\prime} \\
\cdot \frac{2}{\pi w} \frac{T_{q}\left(\frac{x^{\prime}-s}{w / 2}\right)}{\sqrt{1-\left(\frac{x-s}{w / 2}\right)^{2}}} G_{M}^{a s}\left(x, x^{\prime}\right)
\end{gathered}
$$

where $G_{M}^{a s}\left(x, x^{\prime}\right)$ is the spatial domain Green's function of the "asymptotic problem"' (or a related function). The asymptotic problem corresponds to the structure shown in Fig. 1(b). The functions $G_{M}^{a s}\left(x, x^{\prime}\right)$ are tabulated in Table II for the four situations considered in Table I.

The double integrals in (15) could be directly evaluated via the Gauss-Chebyshev quadratures, but the singular behavior of $G_{M}^{a s}\left(x, x^{\prime}\right)$ greatly restricts the computational efficiency. A very large number of quadrature points needs to be used to get acceptable accuracy. In order to overcome this drawback, the logarithmic singularity of $G_{M}^{a s}\left(x, x^{\prime}\right)$ should be extracted and the corresponding convolution integral should be analytically computed. When the strip is close to the side wall $(x=0, a)$, the nearest image line charge significantly affects the convergence of the convolutions. Owing to this, in addition to the singularity taking place in the integration interval, the first two reflected images have been pulled out of the Green's function. The singular terms explicitly considered in the computations, $S\left(x, x^{\prime}\right)$, have also been included in Table II. Let us consider the case in which $x=0$ and $x=a$ are electric walls. The convolutions involving the singular 
TABLE II

asymptotic Spatial Green's Function, $G_{M}^{u s}\left(x, x^{\prime}\right)$, and Analytically Treated Singular Part, $S(x$,

$x^{\prime}$ ), For the Combinations of Lateral Boundary CONditions in Tabl.

\begin{tabular}{|c|c|c|}
\hline & $G_{M}^{a s}\left(x, x^{\prime}\right)$ & $S\left(x, x^{\prime}\right)$ (Singular Terms) \\
\hline $\begin{array}{l}x=0: \text { e.w. } \\
x=a: \text { e.w. }\end{array}$ & $\begin{array}{c}-\frac{1}{2 \pi \epsilon_{e q}}\left\{\ln \left|\sin \left(\frac{\pi}{2 a}\left|x-x^{\prime}\right|\right)\right|\right. \\
\left.-\ln \left[\sin \left(\frac{\pi}{2 a}\left(x+x^{\prime}\right)\right)\right]\right\}\end{array}$ & $\begin{array}{l}-\frac{1}{2 \pi \epsilon_{e q}}\left\{\ln \left|x-x^{\prime}\right|\right. \\
\left.\quad-\ln \left\{\left(2 a-x-x^{\prime}\right)\left(x+x^{\prime}\right)\right]\right\}\end{array}$ \\
\hline $\begin{array}{l}x=0: \text { m.w. } \\
x=a: \text { e.w. }\end{array}$ & $\begin{aligned} &- \frac{1}{2 \pi \epsilon_{e q}}\left\{\ln \left[\tan \left(\frac{\pi}{4 a}\left|x-x^{\prime}\right|\right)\right]\right. \\
&\left.\quad+\ln \left[\tan \left(\frac{\pi}{4 a}\left(x+x^{\prime}\right)\right)\right]\right\}\end{aligned}$ & $\begin{array}{l}-\frac{1}{2 \pi \epsilon_{e q}}\left\{\ln \left[\left|x-x^{\prime}\right|\left(x+x^{\prime}\right)\right]\right. \\
\left.-\ln \left(2 a-x-x^{\prime}\right)\right\}\end{array}$ \\
\hline $\begin{array}{l}x=0: \text { p.b.c. } \\
x=a: \text { p.b.c. }\end{array}$ & $-\frac{1}{2 \pi \epsilon_{e^{4}}} \ln \mid 2 \sin \left(\frac{\pi}{a}\left|x-x^{\prime}\right|\right)$ & $\begin{array}{l}-\frac{1}{2 \pi \epsilon_{e q}}\left\{\ln \left|x-x^{\prime}\right|\right. \\
\left.\quad+\ln \left[\left(a+x-x^{\prime}\right)\left(a-x+x^{\prime}\right)\right]\right\}\end{array}$ \\
\hline $\begin{array}{l}x=0: \mathrm{m} \cdot \mathrm{w} \\
x=a: \mathrm{m} \cdot \mathrm{w} .\end{array}$ & $\begin{array}{l}-\frac{1}{2 \pi \epsilon_{c q}}\left\{\ln \mid 4 \sin \left(\frac{\pi}{a}\left(x+x^{\prime}\right)\right)\right] \\
\quad+\ln \left[\sin \left(\frac{\pi}{a}\left|x-x^{\prime}\right|\right) \mid\right\}\end{array}$ & $\begin{array}{l}-\frac{1}{2 \pi \epsilon_{e q}}\left\{\ln \left|x-x^{\prime}\right|\right. \\
\left.\quad+\ln \left[\left(x+x^{\prime}\right)\left(2 a-x-x^{\prime}\right)\right]\right\}\end{array}$ \\
\hline
\end{tabular}

terms in this case are given by the following expressions:

$$
\begin{aligned}
I_{1}(q, x) & =\int_{\text {strip }} d x^{\prime} \frac{2}{\pi \omega} \frac{T_{q}\left(\frac{x^{\prime}-s}{w / 2}\right)}{\sqrt{1-\left(\frac{x^{\prime}-s}{w / 2}\right)^{2}}} \ln \left|x-x^{\prime}\right| \\
& =\left\{\begin{array}{l}
-T_{q}\left(\frac{x-s}{w / 2}\right) q ; \quad q \geq 1 \\
\ln (\omega / 4) ; \quad q=0
\end{array}\right.
\end{aligned}
$$$$
I_{2}(q, x)=\int_{\text {strip }} d x^{\prime} \frac{2}{\pi w} \frac{T_{q}\left(\frac{x^{\prime}-s}{w / 2}\right)}{\sqrt{1-\left(\frac{x^{\prime}-s}{w / 2}\right)^{2}}} \ln \left(x+x^{\prime}\right)
$$$$
=\left\{\begin{array}{l}
-z_{1}^{q} / q ; \quad q \geq 1 \\
\ln \left(w z_{2} / 4\right) ; \quad q=0
\end{array}\right.
$$

being $z_{1}=\sqrt{u^{2}-1}-u ; \quad z_{2}=\sqrt{u^{2}-1}+u$;

$$
u=\frac{x+s}{w / 2}
$$

$$
\begin{aligned}
I_{3}(q, x)= & \int_{\text {strip }} d x^{\prime} \frac{2}{\pi w} \frac{T_{q}\left(\frac{x^{\prime}-s}{w / 2}\right)}{\sqrt{1-\left(\frac{x^{\prime}-s}{w / 2}\right)^{2}}} \\
& \cdot \ln \left(2 a-x-x^{\prime}\right) \\
= & \left\{\begin{array}{lc}
-(-1)^{q} z_{1}^{q} / q ; & q \geq 1 \\
\ln \left(w z_{2} / 4\right) ; & q=0
\end{array}\right.
\end{aligned}
$$

$$
\text { being } z_{1}=\sqrt{u^{2}-1}-u ; \quad z_{2}=\sqrt{u^{2}-1}+u \text {; }
$$

$$
u=\frac{2 a-x-s}{w / 2}
$$

For the other cases in Table II, we only need to replace $x$ by $a-x$ in (17) and (18) to obtain the necessary integrals. Note that the regularized Green's function, $G_{m}^{a s}\left(x, x^{\prime}\right)-$ $S\left(x, x^{\prime}\right)$, is a very smooth function in the whole integration interval. So, this part of the convolutions can be computed by using very few Gauss-Chebyshev quadrature points. In our programs, we have used $n f+2$ quadrature points where $n f$ is the number of basis functions used in the computations. This choice typically ensures more than eight significant figures to be correct.

Once the convolution integrals have been efficiently evaluated, the inner products in (15) have to be computed. The part of the inner products involving the singular term $I_{1}(q, x)$ can be obtained in closed form:

$$
\begin{gathered}
\int_{\text {strip }} d x \frac{2}{\pi w} \frac{T_{p}\left(\frac{x-s}{w / 2}\right)}{\sqrt{1-\left(\frac{x-s}{w / 2}\right)^{2}}} I_{1}(q, x) \\
=\left\{\begin{array}{cc}
-1 / 2 p & \text { if } p=q \neq 0 \\
0 & \text { if } p \neq q \\
\ln (w / 4) & \text { if } p=q=0
\end{array}\right.
\end{gathered}
$$

Numerical Gauss-Chebyshev quadratures are now used to compute the rest of the inner products. Since no singularities are involved, very high accuracy is achieved by also using $n f+2$ quadrature points in the computations. Only in nonrealistic situations (for example, impracticable 
spacing between lines), the number of points should be increased above that value, as will be commented in the results section. Anyway, even in these cases, convergence is achieved with a reasonable number of quadrature points and the procedure is still very useful. It should be noticed that the convolution integrals are computed just once at the quadrature points corresponding to the inner products. As a consequence of this, the application of a point matching scheme is not essentially more efficient than the Galerkin technique used in this work.

\section{Numerical Results}

The theory developed in the previous two sections has been used to write FORTRAN computer programs which run on a PC computer working at $20 \mathrm{MHz}$. Double precision is used in the computations in order to check the accuracy of the method. It has been found that both procedures provide exactly the same results for the characteristic parameters and the coefficients of the charge distribution (until 16 significant digits equal). The convergence of the series (20), (25), (26) and (27) and the accuracy of the Gauss-Chebyshev quadratures used to compute (15) have been investigated before generating numerical data.

The number of terms to be retained in the computation of the series in the Appendix 1 depends on the aspect ratio $r=(s-w / 2) / w$. This number is related to the distance from the strip to the side walls. In most practical cases this ratio is not too small, and just a few terms are enough to achieve convergence (typically less than 3 terms). More terms need to be added when $r$ is small, that is, when very tightly coupled strips or one strip very close to the side wall are considered. In any case, we have not found serious numerical problems. A typical convergence pattern is shown in Table III. In this table we display the number of terms to be retained $\left(k_{\max }\right)$ to get five significant figures (in the characteristic impedance and the phase velocity) for different values of $r$. The results in Table III were obtained by using $n f=6$ in (2), and the CPU time was not bigger than 0.5 seconds in the worst case (this time includes the computations for the structure without dielectric).

Let us consider now the efficiency of the second method for the computation of the asymptotic tails. As stated above, the part of the convolution integrals in (15) involving the regularized Green's function is computed in all cases by using $n f+2$ quadrature points-that is more than enough. Inner products could require more quadrature points when $r$ is extremely small. Nevertheless, this technique is much less sensitive to the value of $r$. All the results in Table III were obtained with more than 8 digits accuracy by using $n f+2$ quadrature points in the evaluation of the inner products. In the extreme and impractical case $r=0.005,5$ significant figures are obtained by using $n f+14$ quadrature points. In this case the CPU time was 0.7 seconds. In most cases this technique works slightly better than the first one. Anyway, the use of any of these
TABLE III

NUMBER OF TERMS $\left(k_{\max }\right)$ USED IN THE SERIES IN APPENDix A to get Five Correct Digits in the Characteristic Parameters as a FunCtION OF THE ASPECT RATIO $r$

\begin{tabular}{cc}
\hline$r$ & $k_{\max }$ \\
\hline 1.5 & 1 \\
1.0 & 2 \\
0.5 & 3 \\
0.25 & 6 \\
0.1 & 12 \\
0.05 & 24 \\
\hline
\end{tabular}

techniques results in drastic improvement of the SDA scheme. For comparison purposes, it should be emphasized that, if no analytical preprocessing is used, the achievement of a similar accuracy for the capacitance requires adding up $5 \cdot 10^{4}$ Fourier terms (more than 4 minutes of CPU). Prohibitive CPU time should be required for narrower strips. On the contrary we must only add a few spectral terms (typically ten terms are enough) if the techniques in this paper are employed. Moreover, the convergence features of the methods described here do not depend on the strip width but only slightly on the aspect ratio $r$. This is an additional advantage of these procedures over other asymptotic extractions used in the past by the authors (a brief description can be found in the Appendix of [20]). Bessel functions were also approximated in that case by their asymptotic limit together with the Green's function. Good convergence was achieved with that method, but its efficiency decreases with narrow strips and/or if many basis functions are required ( $n f$ is large)-this is the case for wide strips or strong coupling. The techniques in this paper have no limitations with respect to the number of basis functions or the strips width. In particular, the off-diagonal elements of the RitzGalerkin matrix are very accurately computed in contrast to the other techniques. Summing it up, the methods presented here make it possible to obtain much more accurate results for the charge distribution and the characteristic parameters with less CPU time and much higher reliability and generality than the other techniques previously used by us.

The numerical results provided by our programs have been also conveniently checked by comparing with highly accurate data taken from the literature. The propagation parameters obtained for certain structures by means of exact conformal mapping are reproduced with exactness within the accuracy of the computer. However the accuracy of our results is better illustrated by considering the coefficients of the expansion (2). Recently Fikioris et al. [11] reported highly accurate data for the charge distribution on a boxed microstrip. These results were obtained by using very efficient quasi-analytical expressions for the spatial Green's function of a single layer shielded geometry in combination with the Carleman-Vekua regularization method. The methods in our paper need less ana- 
TABLE IV

Coefficients of the Charge Distribution for Single and Coupled with the Ones Reported in [[11], Table I]. Dimensions and ElfCtrical Characteristics are the Same than in that Reference.

\begin{tabular}{llll}
\hline & Fikioris et al. [I]] & Method \#1 & Method \#2 \\
\hline \multicolumn{4}{c}{ (single strip) $(Q=0$ in [11]) } \\
\hline$a_{1} / a_{0}$ & .43027625 & .43030186 & .43030186 \\
$a_{2} / a_{0}$ & .15020234 & .15020671 & .15020671 \\
$a_{3} / a_{0}$ & .06199140 & .06199221 & .06199221 \\
$a_{4} / a_{0}$ & .021819421 & .021820228 & .021820228 \\
$a_{5} / a_{0}$ & .007849483 & .007850208 & .007850208 \\
$a_{6} / a_{0}$ & .002990657 & .00291345 & .002991345 \\
\hline \multicolumn{4}{c}{$($ coupled strips, odd mode) $(Q=-1$ in [11]) } \\
\hline$a_{1} / a_{0}$ & .40354420 & .40354433 & .40354433 \\
$a_{2} / a_{0}$ & .15388507 & .15388538 & .15388538 \\
$a_{3} / a_{0}$ & .05985819 & .05985868 & .05985868 \\
$a_{4} / a_{0}$ & .021669194 & .021669701 & .021669701 \\
$a_{5} / a_{0}$ & .007719048 & .007719641 & .007719641 \\
$a_{6} / a_{0}$ & .002948209 & .002948744 & .002948744 \\
\hline \multicolumn{5}{c}{$($ coupled strips, even mode) $(Q=+1$ in $[11])$} \\
\hline$a_{1} / a_{0}$ & .45614674 & .45619712 & .45619712 \\
$a_{2} / a_{0}$ & .14663990 & .14664847 & .14664847 \\
$a_{3} / a_{0}$ & .06405526 & .06405633 & .06405633 \\
$a_{4} / a_{0}$ & .021964961 & .021966064 & .021966064 \\
$a_{5} / a_{0}$ & .007975686 & .007976541 & .007976541 \\
$a_{6} / a_{0}$ & .003031928 & .0030325802 & .0030325802 \\
\hline
\end{tabular}

lytical effort and are applied to multilayer geometries while keeping very high numerical efficiency. In Table IV we compare our results with the results reported in [[11], Table I] for the case $n f=6$. It can be seen that the agreement is excellent in all cases. Numerical results obtained with the two methods in this paper are exactly equal. One half of second is the typical CPU time to get these results even though the strip is very close to the side wall. The results reported in [[11], Table II] were reproduced by us within 6 or 7 significant figures with CPU times ranging from 0.1 to 0.5 seconds.

Computations using 20 basis functions were achieved in less than 2 seconds on a $20 \mathrm{MHz}$ personal computer (an accuracy of 8 significant figures was imposed to the coefficients of the surface charge expansion). The use of a large number of basis functions is necessary when close proximity or very large strips are involved. As it can be seen, the numerical efficiency, reliability and usefulness of the methods in this paper have been widely proven.

As a final example we present in Table $\mathrm{V}$ the results for the coefficients of the charge distribution and characteristic parameters of a pair of coupled strips on suspended substrate. We have used $n f=10$ for these computations and also $n f=5$. The agreement between the results for the characteristic impedance, $Z$, and the effective permittivity when $n f=5$ and 10 is better than $1 / 10^{7}$. However, $a_{10} / a_{0} \simeq 0.001$ for the odd mode and $\simeq 0.0001$ for the even mode. This means that the number of basis functions that has to be used to compute the charge distribution in an accurate way is bigger than that required to compute the characteristic parameters. This is a natural
TABLE V

Characteristic Parameters (Characteristic Impedance, $Z$, and EFfective Dielectric Constant, $\epsilon_{\text {eff }}$ ) and Coefficients of the CHaRge Distribution EXPANSION (2) For SUSPENDED COUPLED Strips.

DimENSIONS: $a=5 \mathrm{~mm}, h_{1}=3 \mathrm{~mm}, h_{2}=.635 \mathrm{~mm}, h_{3}=5 \mathrm{~mm}, \omega=1$ $\mathrm{mm}, s=0.55 \mathrm{~mm}, \epsilon_{1}=\epsilon_{3}=\epsilon_{0}, \epsilon_{2}=9.6 \epsilon_{0}$.

\begin{tabular}{lcc}
\hline & Odd Mode & Even Mode \\
\hline$a_{1} / a_{0}$ & -0.92016161 & +0.75444525 \\
$a_{2} / a_{0}$ & +0.36842245 & +0.02221162 \\
$a_{3} / a_{0}$ & -0.13634537 & +0.03474569 \\
$a_{4} / a_{0}$ & +0.06394639 & -0.01397521 \\
$a_{5} / a_{0}$ & -0.03201018 & +0.00487788 \\
$a_{6} / a_{0}$ & +0.01585873 & -0.00223805 \\
$a_{7} / a_{0}$ & -0.00788614 & +0.00104146 \\
$a_{8} / a_{0}$ & +0.00396332 & -0.00048860 \\
$a_{9} / a_{0}$ & -0.00200885 & +0.00023665 \\
$a_{10} / a_{0}$ & +0.00102446 & -0.00011651 \\
\hline$Z(n f=5)$ & $30.8366 \Omega$ & $182.8800 \Omega$ \\
$Z(n f=10)$ & $30.8360 \Omega$ & $182.8799 \Omega$ \\
\hline$\epsilon_{\text {eff }}(n f=5)$ & 4.608920 & 2.136619 \\
$\epsilon_{\text {eff }}(n f=10)$ & 4.608930 & 2.136619 \\
\hline
\end{tabular}

consequence of the stationary nature of the capacitance per unit length.

Although this paper has focused its attention on the quasi-TEM model, the method developed here should be also useful in spectral full-wave computations, since the quasi-static limit of the dynamic problem-which is the main factor that limits the convergence-can be analytically treated as explained in this paper. However, the convergence is expected to be worse in the full-wave case, since the dyadic Green's function tends to its asymptotic limit more slowly than the static Green's function. Some improvements are expected to be carried out in this sense in a forthcoming work.

\section{Conclusion}

In this paper we have presented two methods which significantly enhance the numerical convergence of the spectral domain computations of a microstrip line with rectangular boundary conditions and layered substrate. By using these techniques, extremely accurate results for both characteristic parameters and charge distributions can be obtained on a PC computer in a very short CPU time. Virtually exact solutions can be obtained for a variety of single and symmetrically coupled microstrip structures. In spite of the fact that the techniques are very general, they are not as cumbersome as other techniques previously reported in the literature. The extension of these techniques to multistrip systems and full-wave analysis is the object of a future work.

\section{APPENDIX}

In this Appendix, very quickly convergent expressions for the integral in (10) and the integral with respect $\alpha$ of (12) are obtained. In order to evaluate these integrals, the product of modified Bessel functions is expanded as a se- 
ries of powers by using [[19], p. 960]. Acting in this way, we can write:

$$
\begin{array}{r}
\int_{0}^{\infty} d y \frac{I_{p}(\alpha y) I_{q}(\alpha y)}{y \sinh (\pi y)} \cdot\left\{\begin{array}{l}
\cosh [(\pi-c) y] \\
\sinh [(\pi-c) y]
\end{array}\right\} \\
=\sum_{k=0}^{\infty}\left(\frac{a}{2}\right)^{p+q+2 k} F(p, q, k) \\
\cdot \int_{0}^{\infty} y^{p+q+2 k-1} \frac{\left\{\begin{array}{l}
\cosh [(\pi-c) y] \\
\sinh [(\pi-c) y]
\end{array}\right\}}{\sinh (\pi y)}
\end{array}
$$

where

$$
F(p, q, k)=\frac{\Gamma(p+q+2 k+1) \Gamma(p+q+2 k)}{\Gamma(p+q+k+1) \Gamma(q+k+1) \Gamma(p+k+1) \Gamma(k+1)}
$$

where $\Gamma$ is the gamma function. The integrals appearing in the previous expressions are analytically known [[19], pp. 350]:

$$
\int_{0}^{\infty} d x \frac{x^{m}}{\sinh (\pi x)}\left\{\begin{array}{c}
\cosh (\beta x) \\
\sinh (\beta x)
\end{array}\right\}=\frac{1}{2} \frac{d^{m}}{d \beta^{m}} \tan (\beta / 2)
$$

This expression is used for low values of $m(m \leq 9$ which is enough in almost all practical cases). If larger values of $m$ are required to achieve convergence (this rarely occurs), we use an alternative expression [[19], pp. 349]:

$$
\begin{aligned}
& \int_{0}^{\infty} x^{m-1} \frac{\left\{\begin{array}{c}
\cosh (\beta x) \\
\sinh (\beta x)
\end{array}\right\}}{\sinh (\pi x)} d x \\
& =\frac{\Gamma(m)}{(2 \pi)^{m}}\{\zeta(m, q) \pm \zeta(m, 1-q)\} ; \quad q=\frac{1}{2}\left[1-\frac{\beta}{\pi}\right]
\end{aligned}
$$

where $\zeta$ is the Riemann's zeta function. In the case $p=$ $0, q=1$ the following identity [[19], p. 1074, p. 945] must be used:

$$
\zeta(1, q)-\zeta(1,1-q)=\pi \operatorname{coth}(\pi q)
$$

As it was stated in Section III, the series corresponding to $p, q=0$ requires an special treatment. The integral in (12) can be expressed as a power series similar to (20). If we now integrate with respect to $\alpha$ (bearing in mind that the series must give the correct value for $\alpha=0$ ), $S_{0.0}^{\prime}$ can be finally expressed as

$$
\begin{aligned}
S_{0,0}^{\prime}= & \frac{1}{2} \ln [2(1-\cos (c)] \\
& +2 \sum_{k=0}^{\infty} \frac{\Gamma^{2}(2 k+2)}{\Gamma^{2}(k+2) \Gamma^{2}(k+1)(k+1)}\left(\frac{\alpha}{4 \pi}\right)^{2 k+2} \\
& \cdot[\zeta(2 k+2, q)+\zeta(2 k+2,1-q)) ; \quad q=\frac{c}{2 \pi}
\end{aligned}
$$

Similar considerations allow us to compute the integral appearing as the right hand second term in (12). The only difference is that the closed contour to be considered is that shown in Fig. 2(b). The results are

$$
\begin{aligned}
\int_{0}^{\infty} d t & \frac{I_{p}(t) I_{q}(t) \exp \left(-\frac{2 \pi}{\alpha} t\right)}{t\left[1-\exp \left(-\frac{2 \pi}{\alpha}\right)\right]} \\
= & \sum_{k=0}^{\infty} F(p, q, k)\left(\frac{\alpha}{4 \pi}\right)^{p+q+2 k} \zeta(p+q+2 k, 1) .
\end{aligned}
$$

For the particular case $p=0, q=0$, in (8) a derivation/ integration process similar to that applied to get (25) gives place to

$$
\begin{aligned}
S_{0,0}^{\prime \prime}= & \ln (2 / \alpha)+\sum_{k=0}^{\infty} \frac{2 \Gamma^{2}(2 k+2)}{\Gamma^{2}(k+2) \Gamma^{2}(k+1)(k+1)} \\
& \left(\frac{\alpha}{4 \pi}\right)^{2 k+2} \zeta(2 k+2,1) .
\end{aligned}
$$

When a large number of basis functions is used ( $p$ or $q$ is large) or a relatively large number of terms is needed in the computations ( $k$ is large), overflow problems can arise. Owing to this, from a computational point of view, it is preferable to work with the natural logarithms of the factors involved in the general term of the series in this appendix.

\section{REFERENCES}

[1] J. S. Rao and B. N. Das, "Analysis of asymmetric stripline by con formal mapping," IEEE Trans. Microwave Theory Tech., vol. 27 pp. 299-303, Apr. 1979.

[2] H. J. Riblet, "Two limiting values of the capacitance of symmetrical rectangular coaxial strip transmission line," IEEE Trans. Microwave Theory Tech., vol. 29, pp. 661-666, July 1981.

[3] D. Homentcovschi, A. Manolescu, A. M. Manolescu, and L. Kreindler, "An analytical solution for the coupled stripline-like microstrip line problem," IEEE Trans. Microwave Theory Tech., vol. 36. pp. 1002-1007, June 1988.

[4] R. Mittra and T. Itoh, "Charge and potential distributions in shielded striplines," IEEE Trans. Microwave Theory Tech., vol. 18, pp. 149156, Mar. 1970.

[5] J. C. Tippet and D. C. Chang, "Characteristic impedance of a rectangular coaxial line with offset inner conductor," IEEE Trans. Microwave Theory Tech., vol. 26, pp. 876-883, Nov. 1978.

[6] G. M. L. Gladwell, S. Coen, "A Chebyshev approximation method for microstrip problems." IEEE Trans. Microwave Theory Tech., vol 23, pp. 865-870, Nov. 1975

[7] W. C. Chew and J. A. Kong, "Asymptotic eigenequations and ana lytic formulas for the dispersion characteristics of open wide microstrip lines," IEEE Trans. Microwave Theory Tech., vol. 29, pp. 933 941, Sept. 1981

[8] E. F. Kuester and D. C. Chang, "Closed-form expressions for the current or charge distribution on parallel strips or microstrip, " IEEE Trans. Microwave Theory Tech., vol. 28, pp. 254-258, Mar. 1980.

[9] _ - "Theory of dispersion in microstrip of arbitrary width," IEEE Trans. Microwave Theory Tech, vol. 28, pp. 259-265, Mar. 1980. 
[10] B. E. Kretch and R. E. Collin, "Microstrip dispersion including anisotropic substrates," IEEE Trans. Microwave Theory Tech., vol. 35. pp. 710-718. Aug. 1987.

[11] J. G. Fikioris, J. L. Tsalamengas, and G. J. Fikioris, "Exact solutions for shielded printed microstrip lines by the Carleman-Vekua method," IEEE Trans. Microwave Theory Tech., vol. 37, pp. 21 33, Jan. 1989.

[12] J. G. Fikioris, J. L. Tsalamengas, G. J. Fikioris, "Strongly convergent Green's function expansions for rectangularly shielded microstrip lines," IEEE Trans. Microwave Theory Tech.. vol. 36, pp. 1386-1396, Oct. 1988.

[13] K. Uchida, T. Noda, and T. Matsunaga, “New type of spectral-domain analysis of a microstrip line,"' IEEE Trans. Microwave Theor Tech., vol. 37, pp. 947-952, June 1989.

[14] D. Homentcovschi. "An analytical solution to the microstrip line problem,"'IEEE Trans. Microwave Theory Tech., vol. 38, pp. 766769, June 1990.

[15] V. Postoyalko, "Green's function treatment of edge singularities in the quasi-TEM analysis of microstrip," IEEE Trans. Microwave Theory Tech., vol. 34, pp. 1092-1095, Nov. 1986.

[16] N. Faché and D. de Zutter, "Rigorous full-wave space-domain solution for dispersive microstrip lines," IEEE Trans. Microwave Theory Tech., vol. 36, pp. 731-737, Apr. 1988.

[17] F. Medina and M. Horno, "Upper and lower bounds on mode capacitances for a large class of anisotropic multilayered microstrip-like transmission lines," Inst. Elec. Eng. Proc., Pt. H, Microwave, Optics and Antennas, vol. 132, pp. 157-163, June 1985.

[18] M. Horno, F. Mesa, F. Medina, and R. Marqués, "Quasi-TEM analysis of multilayered multiconductor, coplanar structures, with dielectric and magnetic anisotropy including substrate losses," IEEE Trans. Microwave Theory Tech., vol. 38, pp. 1059-1068, Aug. 1990.

[19] I. S. Gradshteyn, I. M. Ryzhik, Table of Integrals, Series and Products. New York: Academic Press, 1980

[20] F. Medina and M. Horno, "Spectral and variational analysis of generalized cylindrical and elliptical strip and microstrip lines," IEEE Trans. Microwave Theory Tech., vol. 38, pp. 1287-1293. Sept. 1990.

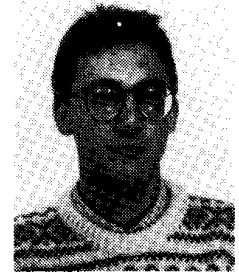

Francisco Medina (M'91) was born in Puerto Real, Cádiz, Spain, on November, 1960. He received the Licenciado degree in September 1983 and the Doctor degree in 1987, both in physics, from the University of Seville, Spain.

He is currently Associate Professor of Electricity and Magnetism in the Department of Electronics and Electromagnetics, University of Seville. $\mathrm{His}$ research deals mainly with analytical and numerical methods for planar structures and multiconductor lines.

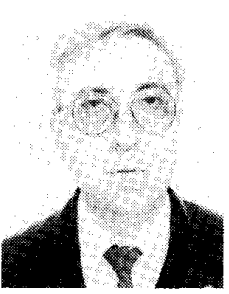

Manuel Horno (M75) was born in Torre de Campo, Jaén. Spain. He received the degree of Licenciado in Physics in June 1969, and the degree of Doctor in Physics in January 1972, both from the University of Seville, Spain.

Since October 1969 he has been with the Department of Electronics and Electromagnetism at the University of Seville, where he became an Assistant Professor in 1970. Associate Professor in 1975 and Professor in 1986. He is a member of Electromagnetism Academy of M.I.T. (Cambridge). His main fields of interest include boundary value problems in electromagnetic theory, wave propagation through anisotropic media, and microwave integrated circuits. He is presently engaged in the analysis of planar transmission lines embedded in anisotropic materials, multiconductor transmission lines, and planar slow-wave structures. 